\title{
Improving User Comfort in Haptic Virtual Environments through Gravity Compensation
}

\author{
Renaud Ott ${ }^{*}$ Mario Gutiérrez ${ }^{*}$ Daniel Thalmann ${ }^{*}$ Frédéric Vexo ${ }^{*}$ \\ (*) Virtual Reality Laboratory (VRLab) \\ École Polytechnique Fédérale de Lausanne (EPFL) \\ Lausanne, Switzerland $\mathrm{CH}-1015$ \\ E-mail: \{renaud.ott, mario.gutierrez, daniel.thalmann, frederic.vexo\}@epfl.ch
}

\begin{abstract}
Our experience with a Haptic Workstation ${ }^{\mathrm{TM}}$ has shown that this device is uncomfortable to use during long sessions. The main reason is the uncomfortable posture of the arms, which must be kept outstretched horizontally while supporting the weight of an exoskeleton. We describe Zero$G$, a real-time weight compensation system aimed at improving user comfort by compensating for the weight of both the exoskeleton and arms (zero gravity illusion). We present experimental results complemented with electro myography measures (EMG) as an indicator of muscular activity/fatigue. Our tests show how Zero-G exerts a positive influence on the reduction of muscular fatigue when using a Haptic Workstation ${ }^{\mathrm{TM}}$.
\end{abstract}

\section{Introduction}

To introduce the work we present in this article we must refer to two apparently unrelated concepts: user comfort and simulation of gravity. Looking for enhancing the user comfort under virtual environments with haptic feedback, we ended up with a gravity simulation system. The rationale is the following: our experience with haptic interfaces using a Haptic Workstation ${ }^{\mathrm{TM}}$ showed that this device is difficult to use during long sessions. One of the main reasons was the muscular fatigue induced by the uncomfortable posture to be adopted while using the workstation (two-arms exoskeleton). The user manipulates virtual objects in a workspace located in front of him. This requires outstretching both arms horizontally, supporting the weight of the arms themselves and the exoskeleton.

The approach we followed consisted on compensating for the weight of the device and the arms. We developed a gravity compensation system called Zero-G: it creates the sensation of working under a zero gravity environment by means of applying vertical-component forces on the wrists.
Next section presents a brief overview of our research work involving a Haptic Workstation ${ }^{\mathrm{TM}}$ (fully immersive virtual environments (VE) with haptic feedback) and our observations concerning the lack of user comfort when using this device.

The rest of the article elaborates on the problem of evaluating comfort and the solution we have implemented. First we show objective measures of the muscular fatigue induced by the use of a Haptic Workstation ${ }^{\mathrm{TM}}$. Then we describe Zero-G, the weight compensation system we developed to overcome this problem. The paper ends by presenting experimental results showing the positive effects of Zero-G on reducing muscular fatigue and creating the illusion of a zero gravity environment.

\section{User comfort on fully immersive VE with haptic feedback}

Our experience with haptic interfaces is based on the development of novel applications using a Haptic Workstation $^{\mathrm{TM}}$. We have proposed the use of this device as the platform for a new kind of user interfaces based on the concept of mediators: virtual objects with haptic feedback that are used to interact within complex environments. Our first experiments showed the feasibility of using virtual interfaces to interact within virtual worlds. In [12] we immersed the user into a virtual world that served as a reconfigurable interface to drive a virtual car. The results were encouraging but we realized the system was not suitable for long sessions. Test users reported some difficulties to manipulate the virtual objects: unfamiliar virtual controls shaped as joysticks suspended in the air. Issues with force-feedback and collision detection were reported as well. But the most important complain was the fatigue experienced after some 10-15 minutes of use.

Our next step was to improve the virtual interface by introducing more familiar control devices (e.g. steering 

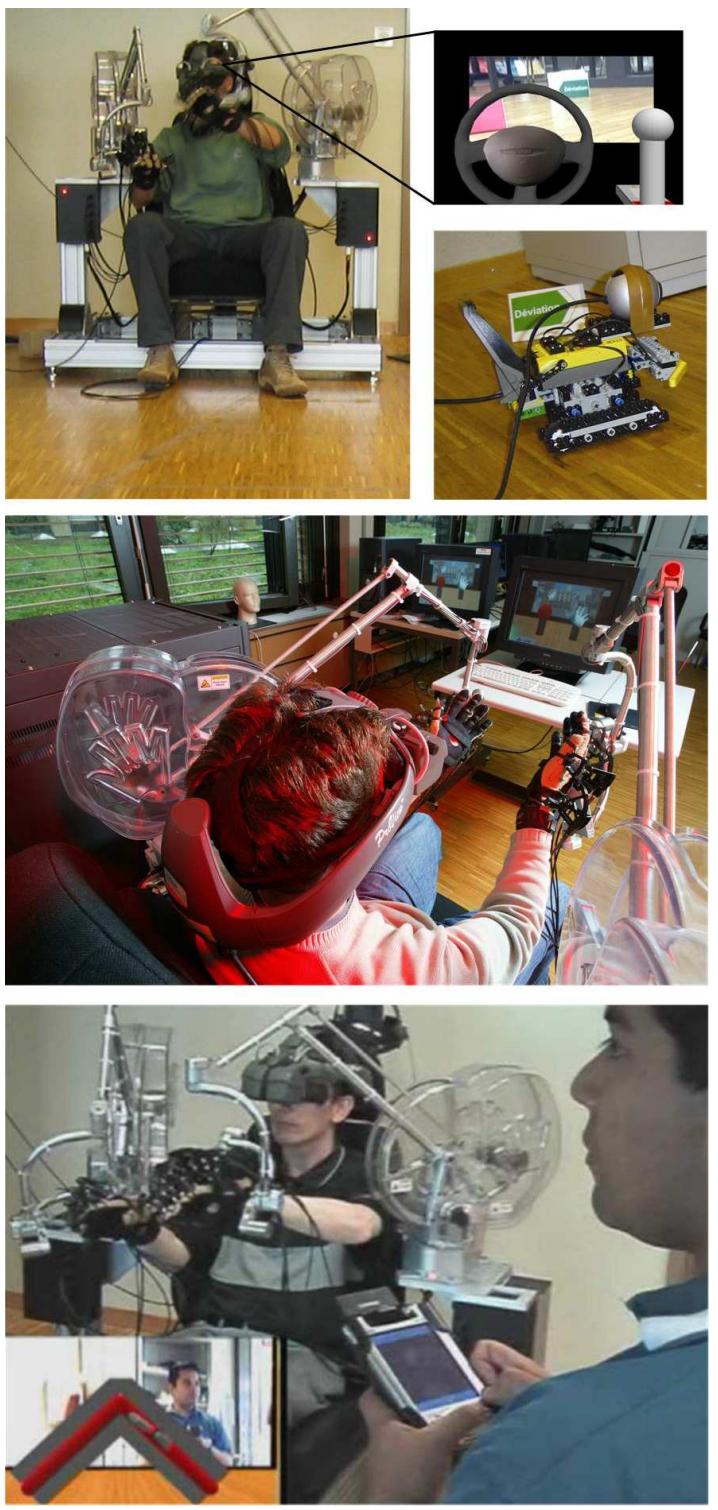

Figure 1. Research using a Haptic Workstation $^{\mathrm{TM}}$. Top: teleoperation of robots; Middle: mediators, driving virtual vehicles; Bottom: telerehabilitation, kinesthetic therapy.

wheels, buttons). This time we tested the system under more realistic conditions: driving a real robot using a virtual interface [7]. We added some extra visual feedback to ease object manipulation within the virtual world: sliders indicating the position of the steering wheel, an speedometer, etc. Users reported the enhanced interface as more maneuverable and easy to learn. However, the lack of comfort remained as an important issue to solve.

A different area of application that we have explored is the tele-rehabilitation. In [6] we described a prototype for providing kinesthetic rehabilitation at distance. The pa- tient sits in the workstation while the therapist can follow the routine, modify it and communicate with him. This test showed a new potential use of the haptic device as a mean to exert controlled forces on the user arms and drive his movements. Tele-therapy consisted on following 3D paths defined by virtual objects in a fully immersive virtual environment. The patient was able to touch the virtual objects and use this feedback to keep the motion of his arms under control, avoiding trembling and enhancing coordination. Nevertheless, long sessions (more than 10 minutes) resulted into tired users complaining about having done too much muscular effort with their arms.

After these experiences our conclusion was that the Haptic Workstation ${ }^{\mathrm{TM}}$ is a rather uncomfortable device. This inconvenience prevents us from obtaining a fair evaluation of its benefits and advantages. The problem of large weight of force-feedback devices has been already identified as one of the main technical drawbacks of haptic technologies [3]. We decided to solve the problem of muscular fatigue by improving the comfort of the Haptic Workstation $^{\mathrm{TM}}$. Next section discusses further about the notion of comfort and how to measure and evaluate it.

\section{Evaluation of comfort}

The goal of this research is to improve user comfort while using a Haptic Workstation ${ }^{\mathrm{TM}}$. This assertion implies that we need to measure the comfort. Unfortunately, this is a subjective notion difficult to quantify. In this section we describe the approach we followed to measure and evaluate it.

Improving the comfort of user interfaces, and in particular the ones used with VR systems is an open area of research. Each case is different and there are many parameters involved in the notion of comfort. In fact, it is difficult to find research focused specifically on this problem. Research is mainly focused on functional studies in impaired patients: upper limb motion analysis in patients with neurological deseases [1]; use of force-feedback to improve GUI interaction tasks [11], [15], [9]; etc.

Most of the studies on haptic interfaces are based on PHANTOM devices. Scientific literature is virtually nonexistent when it comes to evaluating user performance and comfort on a device such as the Haptic Workstation $^{\mathrm{TM}}$.

Improving comfort when using a Haptic Workstation $^{\mathrm{TM}}$ is not like improving it inside a sofa or a car. It is more like enhancing the comfort of a diving-suit. Indeed, the Haptic Workstation ${ }^{\mathrm{TM}}$ was not designed with comfort in mind. After some hands-on experience it is easy to realize how cumbersome and heavy it can be. Using the workstation as shown on figure 2, is equivalent to keeping arms outstretched while holding a $600 \mathrm{~g}$ weight on each wrist. A user can not stay inside more than 


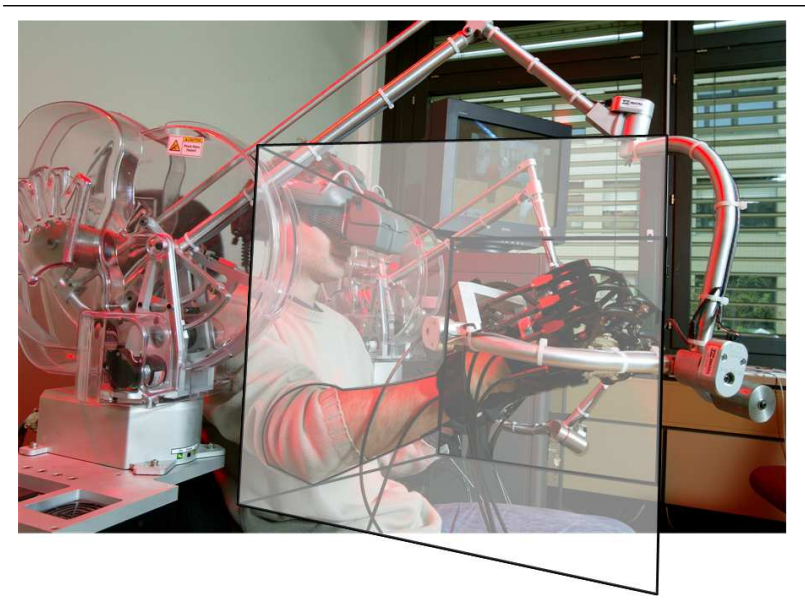

Figure 2. A user driving a virtual car and representation of the cubic working area

half an hour. The notion of comfort is very close to absence of tiredness: if the user were not tired, he could stay probably longer, and we would consider this as a comfortable device. Tiredness is due to muscular activity (which induces fatigue).

Muscular fatigue can be considered as an indicator of tiredness and thus of comfort. The question is how to measure muscular activity over the time. Such measures and experiments are studied and analyzed by physical therapists using bio-feedback techniques. Bio-feedback is a treatment technique in which people are trained to improve their health by using signals from their own bodies [16]. Bio-feedback devices can be used to obtain objective measures of muscular activity and effort, which may serve to deduce the amount of fatigue. Next subsection describes the bio-feedback device we have used to obtain such measures.

\subsection{Bio-feedback measures}

The device we are using is a Physio Recorder S [17], shown in Figure 3. It allows for measuring EMG (ElectroMyoGraphy) signals, among others. The EMG sensor is composed of three electrodes which are used to measure action potentials of one muscle. EMG signals are the manifestation of electrical stimulations, which motor units receive from the Central nervous System, and indicate the activation level of motor units associated with muscle contractions. EMG can be used as a reliable measure of muscular activity and hence is a good indicator of fatigue and comfort. EMG signals are frequently used for providing control commands for prothetic arms [2], [18]. They have also been exploited in studies on user comfort an performance [4].

We used this equipment to evaluate the muscular activity of user's biceps while using the Haptic Workstation ${ }^{\mathrm{TM}}$.

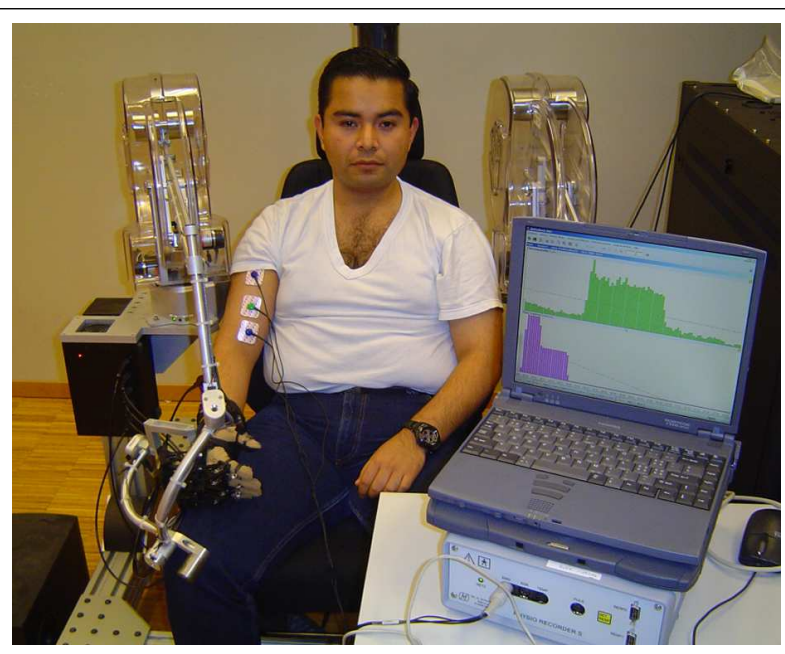

Figure 3. The bio-feedback device evaluating biceps activity of a user

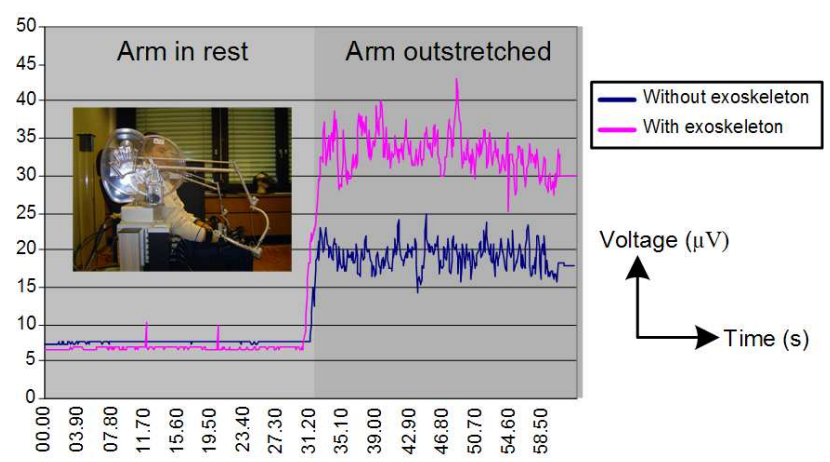

Figure 4. Muscular activity measured with the EMG

Next subsection presents the analysis of the results we obtained.

\subsection{Measuring user comfort}

During thirty seconds, the user kept his arm relaxed, one hand laid down on his knees. This posture would correspond to the minimal biceps activity. Then the user kept his arm outstretched horizontally. This posture was considered as uncomfortable, since it required additional effort on the muscles.

Figure 4 presents the results of measuring muscular activity on user's arm (biceps) in the cases described before. The measured signal increases significantly when posture changes from resting to activity, the voltage is multiplied by up to five times. The graph shows two curves. The lightcolored one represents the muscular effort over time while 
carrying the exoskeleton, whereas the dark-colored one corresponds to tests without it. The $600 \mathrm{~g}$ extra weight from the exoskeleton must be compensated by the arm muscles, increasing their activity -tension- . This was confirmed by the EMG measures: the light curve is above the dark one, indicating an increase on muscular fatigue.

Our goal is to reduce the muscular fatigue indicator, which will traduce into improved comfort and longer sessions using the Haptic Workstation ${ }^{\mathrm{TM}}$. Next subsection describes the approach we have followed to reduce the muscular fatigue.

\subsection{Improving user comfort}

We assume muscular fatigue is mainly due to the extra weight imposed by the exoskeleton of the haptic interface. Thus, our approach consists not only on minimizing this extra weight but also on reducing the weight of the arms. The idea is to put the user under a zero-g virtual environment where muscular fatigue will be minimized, enhancing the sensation of comfort.

A zero-g virtual environment means we need a mechanism to compensate the weight of both exoskeleton and user arms in real-time: at any time the muscular effort of the user will remain under a certain threshold identified as a comfortable amount of muscular activity.

\section{System architecture}

This section will describe the software that allows for calculating and applying an artificial force field which compensates the weight of exoskeleton and arms.

We created a low-level library that allows for controlling each servomotor of the Haptic Workstation ${ }^{\mathrm{TM}}$. We also needed a robust calibration system, since the one provided by the manufacturer is not suitable for our needs (incoherent measures). These components have been designed in order to simplify the implementation of the Zero-G application as described in the previous section.

\subsection{The Haptic Workstation ${ }^{\mathrm{TM}}$}

This subsection deals with the Haptic Workstation $^{\mathrm{TM}}$ devices and how to access them via software.

The Haptic Workstation ${ }^{\mathrm{TM}}$ is composed by four usual devices of virtual reality:

- A pair of CyberGloves ${ }^{\mathrm{TM}}$ used for acquiring hand posture.

- A pair of CyberGrasp ${ }^{\mathrm{TM}}$ used to add force feedback on each fingers.

- A pair of CyberForce ${ }^{\mathrm{TM}}$ which is an exoskeleton used to convey force-feedback to both arms.

- A pair of CyberTrack ${ }^{\mathrm{TM}}$ encapsulated in the CyberForce $^{\mathrm{TM}}$ device to get the position and the orientation of user hands.
Our application does not use the first two devices because we do not need to apply force feedback on the fingers. The hand posture could be considered as a negligible parameter in the force calculation process: the force applied to the hand in a given position does not change significantly when the user moves his fingers. In fact, this application just needs the two last devices. We only have to get hands position with the CyberTrack ${ }^{\mathrm{TM}}$ in order to apply the correct force response with the CyberForce ${ }^{\mathrm{TM}} \mathrm{de}-$ vice.

Usually the Haptic Workstation ${ }^{\mathrm{TM}}$ is programmed with the help of the VHT library [10]. It allows to describe virtual environments with which the user can interact. However in our case we need a general mechanism independent of the virtual environment and the objects inside. Thus VHT is too complicated and high-level for our needs. That's why we have developed our own simple library which contains six basic primitives: connect/disconnect to the Haptic Workstation ${ }^{\mathrm{TM}}$, get left/right hand position, and finally set a force on left/right hand. getPosition() returns a 3D-vector that represents the hand position in the space. setForce() has a 3D-vector parameter that represents the direction along which the hand must be moved. The conversion from this last force vector to a torque on the CyberForce $^{\mathrm{TM}}$ servomotors is calculated by the hardware of the Haptic Workstation ${ }^{\mathrm{TM}}$.

\subsection{The calibration system}

A good calibration procedure is essential to our application. In this subsection we explain why we need it and how the calibration software was designed.

The optical trackers of the CyberForce ${ }^{\mathrm{TM}}$ are very quick and precise, however they still have two drawbacks.

First, they only provide data relative to their position at boot time. It means that the user has to start up the workstation in a given initial position, otherwise the returned values are incoherent. However, the initial position is difficult to reach with precision because we don't have many landmarks. Moreover, the initial position is sometimes lost, forcing the user to reboot the workstation.

The second drawback is that the Haptic Workstation $^{\mathrm{TM}}$ uses different orthogonal coordinate systems for each arm. We require both arms to be in the same space and use orthonormal coordinate systems. In order to use a normal coordinate system, we adopt centimeters as standard units. Moreover, this will simplify further use, in particular when we need to measure the trackers position in real space.

Thus, for each coordinates $x, y$ and $z$, we need to find scaling and translation transformations. The scaling parameter is used to convert coordinate values from units of the Haptic Workstation ${ }^{\mathrm{TM}}$ to centimeters. The translation is used to place right and left arm position in the same space. 


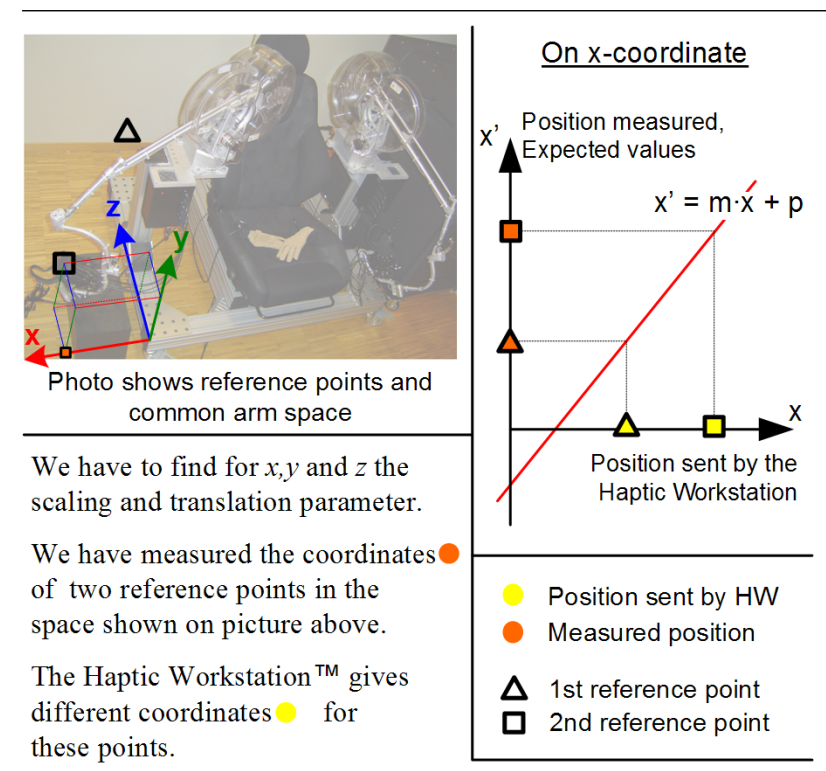

The equation $\left(x^{\prime}=m x+p\right)$ above is easy to calculate having two reference points. It gives the transformation to pass all $x$ coordinates from Haptic Workstation space to the space shown on photography. $\mathrm{m}$ is scaling and $\mathrm{p}$ is translation.

Applying this mechanism for all coordinates $x, y$ and $z$ gives the robust calibration protocol expected for the right arm. It is exactly the same method for the left arm.

\section{Figure 5. Calibration protocol.}

We have chosen two positions of Haptic Workstation $^{\mathrm{TM}}$ exoskeleton that are easy to reproduce with precision. The position of these reference points is measured with a rule and then compared with the coordinate values sent by the workstation to evaluate the six parameters of the transformation (scaling on $x, y, z$, and a 3D-translation), see figure 5.

Finally, to calibrate the Haptic Workstation ${ }^{\mathrm{TM}}$, the user needs to place each arm on two positions (specifying to the computer that these positions are reached e.g. by pressing a button), and then, all returned positions are automatically corrected. This solution to the second drawback eliminates the need of booting the workstation on a fixed position, in consequence it also solves the problem of rebooting the machine when reference data are lost.

\subsection{The anti-gravity software}

The anti-gravity software could be divided into two parts: We first need to calculate the force field that is used to compensate the weight of the exoskeleton and of user's arms. The second part refers to the real-time weight compensation based on the previously calculated force field .

In fact there are two distinct force fields: the first one compensates only the exoskeleton (it is a one-g simulation where people feel the weight of their arms), whereas the second one compensates the exoskeleton and the arms (zero-g simulation). Both force fields are calculated in the same way, as follows.

The force field is a continuous function. We have two solutions to represent it: the analytic method, which gives exact values of the force for each position, but is not suitable for real-time performance; and the discreet method, which returns approximative values in real-time.

Indeed, the equation of the force field is very hard to calculate and to solve in real-time. In [5], the authors present a comparision of different algorithm for gravity compensation of parallel mechanisms (a 5DOF haptic interface). They did not present a real-time controller for such algorithms. The possibility of evaluating these kind of compensation functions in real-time is not guaranteed.

Among the research works focused on the problem of gravity compensation we can also cite the work of Liu, et. al. [13]. The authors developed an estimation and compensation algorithm for the gravity force and static friction of robot motion (6DOF). Nevertheless such a solution is not suitable for our needs. We require a fast way to improve the comfort of the user by helping him to support the weight of both the exoskeleton and his own arms. Moreover the analytic method requires biometrics values (such as bones and soft tissues density, ...) to compensate user arm weight. These are the reasons why we chose a discrete approximation method: it is easier to implement, faster to evaluate and sufficiently precise for our needs.

The first step is to define a working area (see figure 2), a parallelepiped in front of the Haptic Workstation ${ }^{\mathrm{TM}}$, where the weight compensation would be applied. Then, the force field for the exoskeleton and the force field for both exoskeleton and user's arms are consecutively evaluated by the algorithm we will describe.

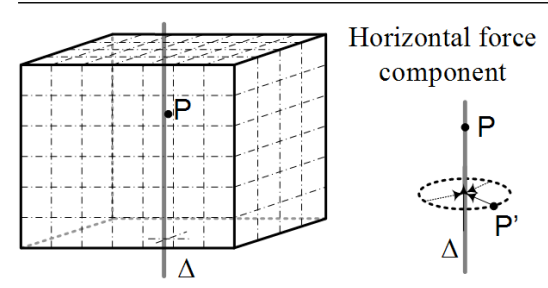

Vertical force component

At each iteration, horizontal force component constrains exoskeleton along $\Delta$. Vertical force component, which is the value searched, is refined to reach the position $P$.

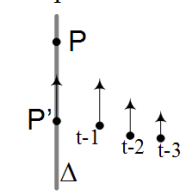
$P$ : position to reach P': hand position
$\Delta$ : vertical line passing trough $\mathrm{P}$

\section{Figure 6. Forces constraining arm position while force field sampling.}

The parallelepiped -working area- is discretized into small cubes and a force is calculated at each of their vertices. Since the force that could nullify the weight is inevitably vertical (horizontal component is negligible), the force vector will be resumed to a scalar. To find the cor- 
rect force value, the algorithm is based on a single loop where the vertical force is refined step after step. At each iteration horizontal forces constrain the position of the exoskeleton's wrist to achieve each pre-defined vertex, as shown in figure 6 . There are three conditions to get out of this loop: the exoskeleton must be immobile, the position must be reached, and these two conditions must stay true during at least two seconds. In fact immobile means "moving slower than a $\epsilon$-speed" (less than $1 \mathrm{~mm} / \mathrm{s}$ ), position reached means "closer than a $\epsilon$-distance" (less than $1 \mathrm{~cm}$ ). Inside the loop, changes are made to the force according the current state, as shown on figure 7 . When all positions have been reached, results are saved in a XML file. The user does not need to interact with the workstation during the sampling of force field. Moreover when the mode that compensates both arms and exoskeleton is selected, a particular user needs to calculate his own force field only once.

\begin{tabular}{|c|c|c|c|c|}
\hline & \multicolumn{3}{|c|}{ Moving State } \\
\hline & & $\begin{array}{l}\text { Going } \\
\text { down }\end{array}$ & $\begin{array}{c}\text { No } \\
\text { Move }\end{array}$ & $\begin{array}{c}\text { Going } \\
\text { Up }\end{array}$ \\
\hline \multirow{3}{*}{ 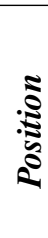 } & Above & $\begin{array}{c}\text { No } \\
\text { change }\end{array}$ & $\begin{array}{c}\text { Low } \\
\text { decrease }\end{array}$ & $\begin{array}{c}\text { High } \\
\text { decrease }\end{array}$ \\
\hline & Same pos. & $\begin{array}{c}\text { Low } \\
\text { increase }\end{array}$ & $\begin{array}{c}\text { No } \\
\text { change }\end{array}$ & $\begin{array}{c}\text { Low } \\
\text { decrease }\end{array}$ \\
\hline & Below & $\begin{array}{c}\text { High } \\
\text { increase }\end{array}$ & $\begin{array}{c}\text { Low } \\
\text { increase }\end{array}$ & $\begin{array}{c}\text { No } \\
\text { change }\end{array}$ \\
\hline
\end{tabular}

Figure 7. Force modification in function of hand states.

The next step is to use these values to nullify the weight. When the hand is in the parallelepiped, the vertical force to be applied depends on the current position. Our approach is to search for the discretized part of the parallelepiped into which the hand is located. Then a weighted mean of the eight vertices of this part gives the force value as shown on figure 8. A 3D-vector with this value for $y$ and 0 for $x$ and $z$ (because $y$ is the vertical axis) is then sent to the setForce () function of the Haptic Workstation ${ }^{\mathrm{TM}}$. The actualization of the forces is done within a frequency of at least $500 \mathrm{H} z$, which corresponds to the Haptic Workstation $^{\mathrm{TM}}$ hardware refresh rate. By this way, we could insure more than enough update of the forces for the user comfort even for quick movements.

In this section we have described the software implementation of the Zero-G application. The next step is to evaluate its performance and validate whether it is useful to improve user's comfort when interacting with virtual environments.

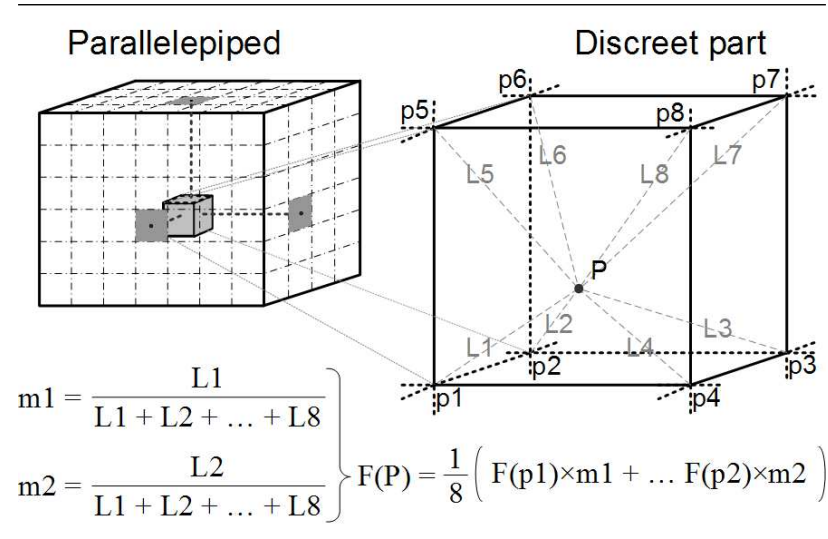

Where $\mathrm{F}(\mathrm{x})$ is force at position $\mathrm{x}, \mathrm{m} \alpha$ is weight of point $\mathrm{p} \alpha$, and $\mathrm{L} \alpha$ is distance between $\mathrm{p} \alpha$ and $\mathrm{P}$.

\section{Figure 8. Getting the force at each position of the space}

\section{Test Results}

This section introduces the tests designed to validate whether Zero-G improves user comfort. First of all, we need to guarantee that the calibration system is efficient. Then, we are going to test the Zero-G software to ensure it does not disturb the performance of the workstation. Finally, when both precedent conditions will be reached, we will need to show that this system is useful to reduce user tiredness when using the Haptic Workstation ${ }^{\mathrm{TM}}$ during a long period.

\subsection{Calibration system}

In the precedent section we have explained that the calibration system is absolutely necessary to the Haptic Workstation $^{\mathrm{TM}}$ : Coordinate system of the Haptic Workstation $^{\mathrm{TM}}$ is not static, and our Zero-G software stores the calculated force field according to a particular coordinate system. Thus we need to verify that it works under different conditions in which we use the Haptic Workstation ${ }^{\mathrm{TM}}$.

Firstly, we have tested our system by placing arms in arbitrary positions and comparing corrected and measured values. In the worst case, the gap between both values is less than one centimeter. This is enough for the Zero-G software (force field does not change significantly in one centimeter).

Secondly, we need to prove that the calibration software is robust. It means that when the Haptic Workstation ${ }^{\mathrm{TM}}$ resets its initial tracker values, a second calibration process may solve the problem. To test that, we use the Device Control Unit (DCU), a software provided by Immersion ${ }^{\mathrm{TM}}$, that allows to manually change the initial tracker values. Our tests showed that our calibration software effectively 
recovers the correct tracker values avoiding the need to reboot the workstation to have correct position data.

\subsection{Zero Gravity software}

In order to test the Zero-G software, we need to calculate the force field at least once. This method is a good approach to compensate for the weight of the CyberForce ${ }^{\mathrm{TM}}$. Then we will show that it works when compensating the user's arm too.

First of all, user needs to define the parallelepiped into which the force field is calculated and its number of subdivisions. Then the process runs automatically without need of user supervision. The CyberForce ${ }^{\mathrm{TM}}$ exoskeleton must reach all positions. To avoid damages on the workstation, the speed of the CyberForce ${ }^{\mathrm{TM}}$ is slow (few millimeters per second), thus it takes a long time to finish, at least 2 hours to reach $7 \times 7 \times 7=343$ positions. Considering that there is no user during this step, the time is not really a problem. Once the force field is saved, the easiest way to test it is to place the exoskeleton at many positions into the parallelepiped and to release it gently. The result is that the exoskeleton doesn't move: exactly the behavior we were expecting. Moreover, when the user touches the exoskeleton he can feel that there is no force constraining the movement. It means the force is minimal. We have tested experimentally that the minimal weight required to break equilibrium (move down the exoskeleton) is of approximately $5 g$.

The second step is to test the software while a user is seated into the Haptic Workstation ${ }^{\mathrm{TM}}$. The procedure is exactly the same as the precedent. Fortunately, during the force field calculation, the user can help the CyberForce $^{\mathrm{TM}}$ to reach the position (reducing the time to complete calculation), but he has to be careful to stop moving before the algorithm starts to evaluate the force. A visual signal is provided into the user interface to tell him when he must be relaxed. After the force field calculation, the user can start to use it. But there is no perfect test to see if it works by telling the user to extend his arm and see if it moves: this because it's hard to be conscious that we do not exert any muscular effort. Thus, we chose to use a bio-feedback device, in order to have an objective measure of muscular effort. For the moment, all we have is the impression that the arm feels lighter.

\subsection{Bio-feedback tests results}

The bio-feedback device allows to quantify the muscular effort. Thus we have designed a test protocol for zero-g and one-g where the user must keep his arm outstretched during few seconds.

The results are presented on figure 9. The objective was to test the influence of one-g (compensation for the exoskeleton weight) and zero-g (compensation for the weight of both the exoskeleton and user's arm) on the muscular fatigue indicator. Each test session is divided into three thirty-seconds-steps. During the first step we activate one$\mathrm{g} /$ zero-g and the user keeps his arm in the parallelepiped where force field is applied. Then, during the next thirty seconds, we deactivate one-g/zero-g and the user has to keep his arm in the same posture. The last step is the reference measure that we will compare to the first one. In the case of one-g, it corresponds to the posture with the outstretched arm that one-g should reproduce: compensating for the weight of the exoskeleton. In zero-g, we compare it to the posture with outstretched arm, but resting on a table, which corresponds to the arm posture under zero-g: compensating for the weight of both the exoskeleton and the arm. Peaks that appear at around thirty seconds and one minute should not be considered because they correspond to the movement of the user changing the posture of his arm.
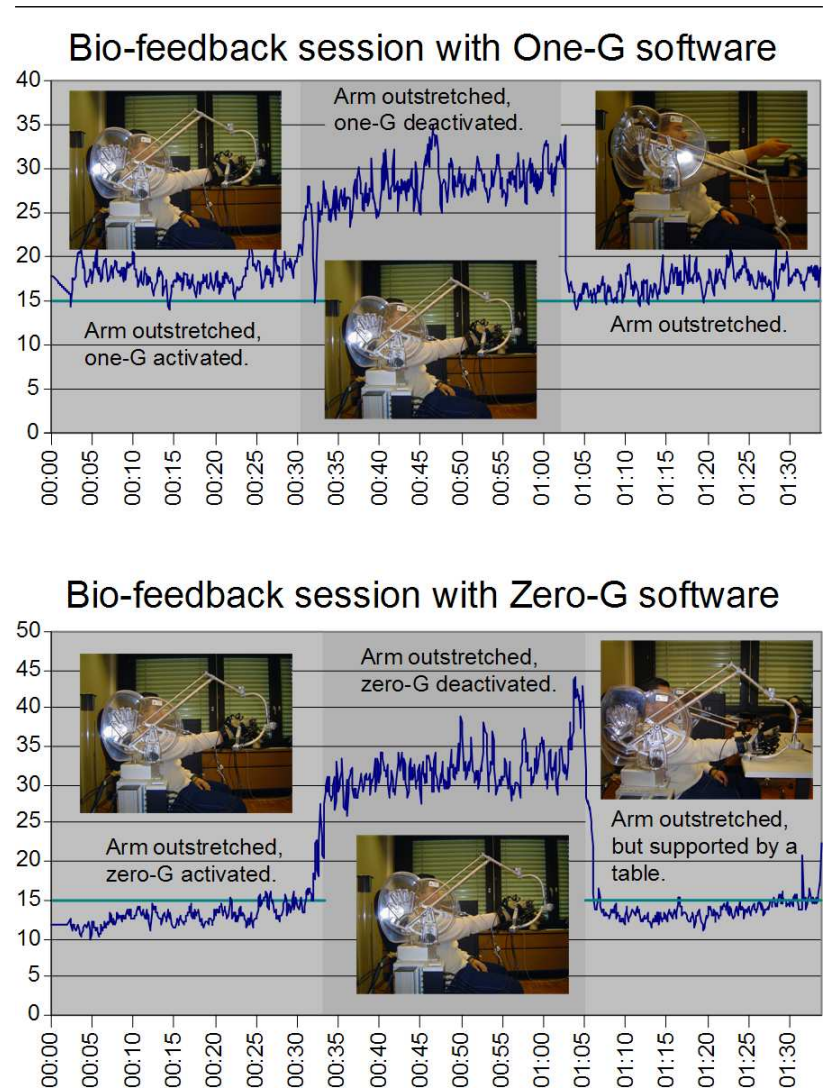

\section{Figure 9. Effect of gravity compensation on muscular activity}

Values returned by the EMG seem coherent. Indeed, muscular effort is less for zero-g than for one-g. Moreover, the reference measure for one-g (last $30 s$ ) was already calculated and presented on figure 4 . We remark that there are almost same values (between 15 and $20 \mu \mathrm{V}$ ). 
Results for one-g and zero-g weight compensation are satisfactory. We could see that user's muscular effort with weight compensation (first part) is equivalent to the muscular effort for keeping arm outstretched and resting on a table for the case of zero-g (last part). It means that oneg compensates for the exoskeleton weight, simulating the earth's gravity, while zero-g effectively eliminates all gravity effects.

These results confirm what we have seen in the precedent subsection: one-g and zero-g could really decrease muscular fatigue, improving user comfort without constraining motion.

\section{Conclusion and further work}

In this article, we have presented Zero-G, a software for gravity compensation in haptic environments. Using a Haptic Workstation ${ }^{\mathrm{TM}}$ (two-arms exoskeleton) for more than 15 minutes was impossible due to the lack of comfort: muscular fatigue on the arms. The weight of the exoskeleton and the uncomfortable posture of the arms were identified as the main sources of fatigue. We used biofeedback techniques (EMG) to measure the muscular activity of the arms in different postures with and without the exoskeleton and using different levels of gravity compensation. Gravity compensation was achieved by means of Zero-G, a software designed to prevent the user from carrying the exoskeleton. Our software compensates for the exoskeleton weight by means of applying a force field (vertical forces) on the wrists. We described a discreet method able to exert weight compensation in real-time. Our method is based on pre-calculation of force fields and an improved calibration algorithm. EMG measures demonstrated that Zero-G effectively reduces the muscular effort required to work while wearing the exoskeleton. Our measures show how Zero-G improves the user comfort attaining muscular activity levels comparable to those obtained for a resting posture (minimal effort). Zero-G gives more realism to the virtual environments, because it partially removes the sensation of wearing an exoskeleton. It allows also to compensate for the weight of the user's arms, giving the illusion of zero gravity.

Zero-G can be used in a variety of applications. Altered gravity environments or controlled variations of the gravitational field can be useful to convey information through additional dimensions.

The work presented in [14] shows how haptic technologies can provide another dimension of information critical to the efficient visualization of data: application to a network intrusion detection system with visual analyzer. In this work, haptic simulation, termed "gravity waves", is used as an unobtrusive means of providing significance to a single node or a group of nodes. As further work we consider developing an interaction framework that will exploit such additional dimensions to convey information in the form of haptic simulation of altered gravity and other physical phenomena.

Studies such as the one reported in [8] show the benefits of incorporating force feedback gravity wells into GUIs as a method of assisting users in performing "pointand-click" tasks. This has been particularly useful for impaired users. Following these results, we believe our system can be helpful not only as an advanced user interface, but also as a system for physical therapy and rehabilitation. In [6] we demonstrated the potential of the Haptic Workstation $^{\mathrm{TM}}$ as a telerehabilitation tool. We plan to incorporate Zero-G to increase its applications range, providing novel kinds of therapy through controlled gravity fields.

\section{Acknowledgments}

This work has been supported by the Swiss Federal Office for Education and Science in the framework of the European IST- Network of Excellence ENACTIVE.

\section{References}

[1] A. Bardorfer, M. Munih, A. Zupan, and A. Primozic. Upper limb motion analysis using haptic interface. IEEE/ASME Transactions on Mechatronics, 6(3):253-260, 2001.

[2] N. Bu, O. Fukuda, and T. Tsuji. Emg-based motion discrimination using a novel recurrent neural network. J. Intell. Inf. Syst., 21(2):113-126, 2003.

[3] G. Burdea. Haptics issues in virtual environments. In Proceedings of Computer Graphics International, pages 295$302,2000$.

[4] A. E. Çakir, G. Çakir, T. Müller, and P. Unema. The trackpad: a study on user comfort and performance. In Conference companion on Human factors in computing systems, pages 246-247. ACM Press, 1995.

[5] D. Checcacci, E. Sotgiu, A. Frisoli, C. Avizzano, and M. Bergamasco. Gravity compensation algorithms for parallel haptic interface. In Proceedings of IEEE International Workshop on Robot and Human Interactive Communication, Berlin, Germany, pages 140-145, 2002.

[6] M. Gutierrez, P. Lemoine, D. Thalmann, and F. Vexo. Telerehabilitation: Controlling haptic virtual environments through handheld interfaces. In Proceedings of ACM Symposium on Virtual Reality Software and Technology (VRST 2004), pages 195-200, 2004.

[7] M. Gutierrez, R. Ott, D. Thalmann, and F. Vexo. Mediators: Virtual haptic interfaces for tele-operated robots. In Proceedings of the 13th IEEE International Workshop on Robot and Human Interactive Communication (RO-MAN 2004), pages 515-520, 2004.

[8] F. Hwang, S. Keates, P. Langdon, and P. J. Clarkson. Multiple haptic targets for motion-impaired computer users. In Proceedings of the conference on Human factors in computing systems, pages 41-48. ACM Press, 2003. 
[9] F. Hwang, S. Keates, P. Langdon, P. J. Clarkson, and P. Robinson. Perception and haptics: towards more accessible computers for motion-impaired users. In Proceedings of the 2001 workshop on Percetive user interfaces, pages 1-9. ACM Press, 2001.

[10] Immersion Corporation ${ }^{\mathrm{TM}}$. Virtual hand sdk datasheet. http://www.immersion.com/3d/products/virtualhand_sdk.php.

[11] S. Keates, F. Hwang, P. Langdon, P. J. Clarkson, and P. Robinson. Cursor measures for motion-impaired computer users. In Proceedings of the fifth international ACM conference on Assistive technologies, pages 135-142. ACM Press, 2002.

[12] P. Lemoine, M. Gutierrez, F. Vexo, and D. Thalmann. Mediators: Virtual interfaces with haptic feedback. In Proceedings of EuroHaptics 2004, 5th-7th June, Munich, Germany, pages 68-73, 2004.

[13] M. Liu and N. H. Quach. Estimation and compensation of gravity and friction forces for robot arms: Theory and experiments. J. Intell. Robotics Syst., 31(4):339-354, 2001.

[14] K. Nyarko, T. Capers, C. Scott, and K. Ladeji-Osias. Network intrusion visualization with niva, an intrusion detection visual analyzer with haptic integration. In Proceedings of the 10th Symposium on Haptic Interfaces for Virtual Environment and Teleoperator Systems, page 277. IEEE Computer Society, 2002.

[15] S. Patomäki, R. Raisamo, J. Salo, V. Pasto, and A. Hippula. Experiences on haptic interfaces for visually impaired young children. In Proceedings of the 6th international conference on Multimodal interfaces, pages 281-288. ACM Press, 2004.

[16] B. Runck. What is Biofeedback? DHHS Publication No (ADM) 83-1273, Arizona Behavioral Health Associates, P.C. http://www.psychotherapy.com/bio.html.

[17] Schuhfried GmbH. Physiorecorder. http://www.schuhfried.co.at.

[18] A. Soares, A. Andrade, E. Lamounier, and R. Carrijo. The development of a virtual myoelectric prosthesis controlled by an emg pattern recognition system based on neural networks. J. Intell. Inf. Syst., 21(2):127-141, 2003. 